\title{
Porous Characteristics of Three-Dimensional Disordered Graphene Networks
}

\author{
YongChao Wang, YinBo Zhu *(D) and HengAn Wu
}

check for

updates

Citation: Wang, Y.; Zhu, Y.; Wu, H Porous Characteristics of Three-Dimensional Disordered Graphene Networks. Crystals 2021, 11, 127. https://doi.org/10.3390/ cryst11020127

Academic Editor: Mahdi Hamidi Received: 27 December 2020

Accepted: 24 January 2021

Published: 28 January 2021

Publisher's Note: MDPI stays neutral with regard to jurisdictional claims in published maps and institutional affiliations.

Copyright: (C) 2021 by the authors Licensee MDPI, Basel, Switzerland This article is an open access article distributed under the terms and conditions of the Creative Commons Attribution (CC BY) license (https:// creativecommons.org/licenses/by/ $4.0 /)$
CAS Key Laboratory of Mechanical Behavior and Design of Materials, Department of Modern Mechanics, CAS Center for Excellence in Complex System Mechanics, University of Science and Technology of China, Hefei 230027, China; wyc1994@mail.ustc.edu.cn (Y.W.); wuha@ustc.edu.cn (H.W.)

* Correspondence: zhuyinbo@ustc.edu.cn

Abstract: The porous characteristics of disordered carbons are critical factors to their performance on hydrogen storage and electrochemical capacitors. Even though the porous information can be estimated indirectly by gas adsorption experiments, it is still hard to directly characterize the porous morphology considering the complex 3D connectivity. To this end, we construct full-atom disordered graphene networks (DGNs) by mimicking the chlorination process of carbide-derived carbons using annealing-MD simulations, which could model the structure of disordered carbons at the atomic scale. The porous characteristics, including pore volume, pore size distribution (PSD), and specific surface area (SSA), were then computed from the coordinates of carbon atoms. From the evolution of structural features, pores grow dramatically during the formation of polyaromatic fragments and sequent disordered framework. Then structure is further graphitized while the PSD shows little change. For the obtained DGNs, the porosity, pore size, and SSA increase with decreasing density. Furthermore, SSA tends to saturate in the low-density range. The DGNs annealed at low temperatures exhibit larger SSA than high-temperature DGNs because of the abundant free edges.

Keywords: graphene; disordered graphene networks; porous characteristics

\section{Introduction}

The large variety of carbon materials with different chemical and structural forms are used in many fields, such as hydrogen storage devices [1], filtering membranes [2], catalysis [3], and supercapacitors [4]. Even though low-dimensional carbon-based materials, including graphene [5], carbon nanotubes [6], and fullerene [7] demonstrate unique chemical and physical properties because of their ordering and reduced size [8], disordered carbons are more widely used because of less cost and widespread resources. Disordered carbons, including activated carbons [9], carbide-derived carbons (CDCs) [10], flash graphene [11], pyrolytic carbons [12], etc., can be produced using different strategies, and the products exhibit different porosity and structures. Active carbons are usually made from carbonaceous source materials (wood, bamboo, coconut husk, etc.) through an activation process by hot gases or certain chemicals [13]. The CDCs have been synthesized from different metal carbides by etching noncarbon species in various temperatures $[14,15]$. Recently, flash graphene (FG) are obtained by flash joule heating of inexpensive carbon sources with low energy consumption $[11,16]$. These disordered carbon materials usually exhibit local ordering of a honeycomb in-plane structure, but they are noncrystalline on a large scale [17-19]. So, they can be regarded as 3D disordered graphene networks (DGNs) with varying graphitization levels and densities.

The porosity and specific surface area (SSA) are the critical factors that influence the performance of DGNs in gas adsorption applications [20,21]. Cabria and coworkers predicted that spherical pores' optimal radius lies in the range between 8.5 and $10.7 \AA$ [22]. In experiments, argon sorption analysis is often used to calculate SSA, pore size distributions (PSD), and pore volumes [23]. Many groups reported that the pore size 
in CDCs could be tailored by changing the chlorination temperature [10,15]. Furthermore, the less graphitized networks can store more hydrogen than highly graphitized ones [24].

Many simulation methods have been proposed to reconstruct the structure of DGNs, providing models for studying of the porous properties. The reverse Monte Carlo (RMC) method and Molecular Dynamics (MD) method are common ways to construct the model. The RMC method uses structure features obtained in experiments as inputs and transforms the current model into new configurations that will reduce the difference between the features of the simulated model and the input data $[22,25,26]$, while the MD method mimics the processing of the material and don't need any inputted experimental features. Inspired by the formation of CDCs from metal carbides, quenching-MD and annealing-MD are often performed to transform an FCC lattice carbon to a disordered graphene network [27]. The quenching-MD simulations use decreasing temperature settings, which can hardly be associated with experimental conditions [28-30]. Tomas and coworkers recently presented an Arrhenius framework in annealing-MD simulations that can associate the short simulation time and high simulation temperature with experimental chlorination time and temperature [31,32]. They also compared the performance of six commonly used interatomic potentials on simulating disordered carbons and found that the environment-dependent interaction potential (EDIP) by Marks [33] can better reproduce the graphitization level and stacked structures [34].

The previous annealing-MD simulations mainly focused on improving the model to be comparable with experiments in terms of coordination number, carbon rings, and pair distribution functions. However, the porous properties, which are essential to applications, are either ignored [34] or characterized using small models with only thousands of atoms [27]. The limited system size precludes the possibility of large pores. Furthermore, the previous works did not distinguish whether the pore is open or closed. In this work, we followed the Arrhenius framework and modified the annealing-MD strategy to investigate the porous features of DGNs using models containing over 100,000 atoms. From the long-time and large-scale annealing simulations, DGNs with varying densities and graphitization levels were obtained. Pores are detected from the coordinates of carbon atoms. In addition, we perform percolation analysis to distinguish the open pores, from which the PSD and SSA can be computed. We analyzed the formation and evolution of pores in different annealing stage. Furthermore, we also discussed the influence of density and annealing temperatures, which may be potential measures to control the porous properties of DGNs.

\section{Methods}

\subsection{Annealing-MD Simulations}

Inspired by the formation process of CDCs [15], we used the annealing-MD simulations to construct DGNs at different conditions. During the experimental preparation of $\mathrm{CDCs}$, metal atoms are removed from the carbide lattice by high-temperature chlorination treatment, leaving a pure carbon material. In the simulated reconstruction, we ignored the influence of metal atoms and only considered the carbon atoms in the lattice. The starting model containing 108,000 atoms was obtained by replicating an FCC lattice of $1.0 \mathrm{~g} / \mathrm{cm}^{3}$. The atoms were assigned initial velocities corresponding to $300 \mathrm{~K}$, and the temperature was maintained in the canonical (NVT) ensemble for $10 \mathrm{ps}$ to reduce the energy. During this procedure, the density can be adjusted by changing the box size and remapping the atoms. After that, the temperature was raised to the target annealing temperature in another $10 \mathrm{ps}$, and annealing simulations were performed for a very long time of $2000 \mathrm{ps}$. The chlorination temperature used in experiments ranges from 600 to $1200^{\circ} \mathrm{C}$, and the process lasts for several hours [35]. However, the time scale of MD simulations is much smaller than that of experiments. To achieve a similar graphitization level with experiments, we should raise the simulated temperature to accelerate the annealing process. Tomas and coworkers suggested an Arrhenius framework to correlate the experimental temperatures and simulation temperatures [31]. We followed this framework and used target temperatures of $1000 \mathrm{~K}$ to $4000 \mathrm{~K}$ to mimic the experimental conditions (from 600 to $1200{ }^{\circ} \mathrm{C}$ ). 
After a long time of annealing simulations under NVT ensemble, DNGs formed, but the temperature and pressure were not suitable for further characterization. So, we lowered the system temperature to $300 \mathrm{~K}$ and changed the box size to meet zero pressure under the isothermal-isobaric (NPT) ensemble. After annealing, there were free atoms and gaseous clusters left in the system. Cluster analysis were performed, and we can easily distinguish the small clusters from those spanning the simulation box. These gaseous clusters were far smaller, containing less than ten atoms. So, these atoms were not considered part of the adsorbent. We followed the treatment in the literature and removed them.

In the annealing simulations, the carbon-carbon interactions were simulated by the EDIP potential provided by Marks [33]. This potential has been proved reliable to reproduce the graphitization behavior of carbons in a wide range of densities [34]. However, a drawback is that the long-range term was not included in the EDIP potential. To reduce the impact of this factor, we performed further relaxation under NPT ensemble for 200 ps, using the Adaptive Intermolecular Reactive Empirical Bond Order potential (AIREBO) [36], which includes an L-J term describing the attractive forces between layers [37-39]. After that, the system's energy minimization was performed to get the optimized atomic configuration of DGNs [40].

The MD simulations were iterated using the code of LAMMPS [41]. We obtained a series of DGNs with varying densities and annealing temperatures. As a representative, the DGN of $1.0 \mathrm{~g} / \mathrm{cm}^{3}$ annealed at $4000 \mathrm{~K}$ was selected to investigate the formation of structures and pores during the annealing process. The coordination, from which $s p^{2}$ and $s p^{3}$ atoms can be identified, was computed by the embedded command in LAMMPS. The carbon rings were characterized using homemade code based on the "shortest-path" algorithm [42]. To calculate the edge length, we first identified edge atoms. Second, cluster analysis was applied to these atoms. Third, bond lengths were computed in each cluster and were summed up as the total edge length. The atomic configurations were rendered in OVITO [43].

\subsection{Porosity Characterization}

POREBLAZER is a set of computational tools for the porosity characterization of porous materials. Its code has been released by Prof. Sarkisov and coworkers [44]. We have improved some algorithms in the code to make it efficient to calculate the porous properties of materials with a side length of more than twenty nanometers. The computation started with gridding the simulation cell into cubes of $0.5 \AA$ in length. Then each cube was tested whether it was occupied by the carbon atoms with a diameter $\sigma_{C}=3.4 \AA$. For cubes that were not occupied by carbon atoms, we used a probe of a hydrogen atom with a diameter $\sigma_{\mathrm{p}}=2.65 \AA$ to test whether the probe can occupy the cube without overlapping with the carbon atom. We chose the L-J parameter for $\mathrm{H}-\mathrm{H}$ interactions as the probe diameter because this parameter has been used to simulate hydrogen adsorption in carbon materials [45]. If the cube could be occupied by the probe, it was defined as a portion of pores. Furthermore, the total volume of these cubes can be computed, representing the total pore volume in the material. We performed percolation analysis on the pores. Pores were replicated by $3 \times 3 \times 3$ along the periodic boundary. After that, cluster analysis was performed in the nonperiodic boundary condition. If a cluster can connect two opposite surfaces of the replicated box, the prereplicating cubes in the cluster are considered to belong to the open pores. The centers of porous cubes were imported to OVITO to construct the surface mesh, which can be further rendered as the morphology of pores.

The surface area of a DGN is the sum of accessible surface area for all carbon atoms. That is

$$
A=\sum_{i} A_{i}
$$

For each carbon atom, to calculate $A_{i}$, a sphere was created at the center with a radius $R=\left(\sigma_{\mathrm{C}}+\sigma_{\mathrm{p}}\right) / 2$. Then five hundred points were randomly selected on the sphere surface, and we tested whether the point was located in one of the accessible cubes. From the 
number of the accessible points $N_{i}$, the accessible surface area of the carbon atom can be estimated as

$$
A_{i}=\frac{N_{i}}{500} S
$$

where $S$ is the total surface area of the sphere.

To calculate the pore size distribution of the DGNs, first, the estimated range of pore size was divided into bins in $2 \AA$. Second, ten thousand points were randomly selected in the simulation box. Third, for each point, if it was located in one of the accessible cubes, the largest empty sphere that contains this point was detected. Then, the values of the bin corresponding to the size of this sphere and the smaller bins were added by one. This procedure resulted in the cumulative pore size function. Finally, the PSD can be calculated by numerical differentiation of this function.

\section{Results and Discussion}

We simulated the evolution of nanoporous disorders graphene networks from a precursor with FCC carbon lattice. In the beginning, the carbon atoms were set an ensemble of velocities corresponding to $300 \mathrm{~K}$, followed by a thermal relaxation of $10 \mathrm{ps}$. The initial configuration of individual carbon atoms was highly unstable, and it collapsed to a network of carbon chains quickly. Then the temperature was raised to the target annealing temperature in another 10 ps and be kept constant until 2 ns. We performed percolation analysis on pores to test whether the pore is open or closed. Furthermore, surface area is calculated only for the open pores. As a representative, the DGN of $1.0 \mathrm{~g} / \mathrm{cm}^{3}$ annealed at $4000 \mathrm{~K}$ was investigated in detail to obtain insights into the formation and evolution of porous structures. According to structural evolution, three stages can be identified.

In the first stage (from 10 ps to $20 \mathrm{ps)}$ ), as the temperature began to rise, the number of carbon rings increased dramatically. We identified the edges of the fragments composed of more than three rings to help to understand how the pores formed. At $10 \mathrm{ps}$, the start point of the annealing process, the structure was a network of carbon chains. The atomic structure in Figure 1c shows a small amount of separated small fragments of rings. Overall, carbon atoms distributed sparsely and evenly without certain gathering, preventing the probe atom from passing through. So, at this point, there were no open pores, and the accessible surface area was zero. As time goes, carbon atoms gathered to form more rings, and the size of $s p^{2}$ dominated fragments increased, resulting in the formation of local pores. At 17 ps, several local pores connected to form open pores, and the volume of open pores increased sharply during the following annealing process. Benefit from the increasing open pore volume and edges, the accessible surface area also increased during this stage. As shown in Figure 1d, at the end of this stage, the structure transformed into a network of polyaromatic fragments with abundant edges.

In the second stage (from 20 ps to 100 ps), the polyaromatic fragments merged into a disordered framework with interconnected $s p^{2}$ dominated layers. The volume of open pores continued to increase as the aggregation of small fragments allowed more pores connected and accessible. This assembling process involves the merging of edges, so the total edge length declined. The accessible surface area, which was affected by both the open pore volume and the edges, increased until 30 ps. Then, it decreased slightly with annealing time. At the end of this stage, a framework of graphene-like layers formed, which were mainly composed of hexagonal rings. There were also some nonhexagonal rings as abundant defects in the disordered framework. 
(a)

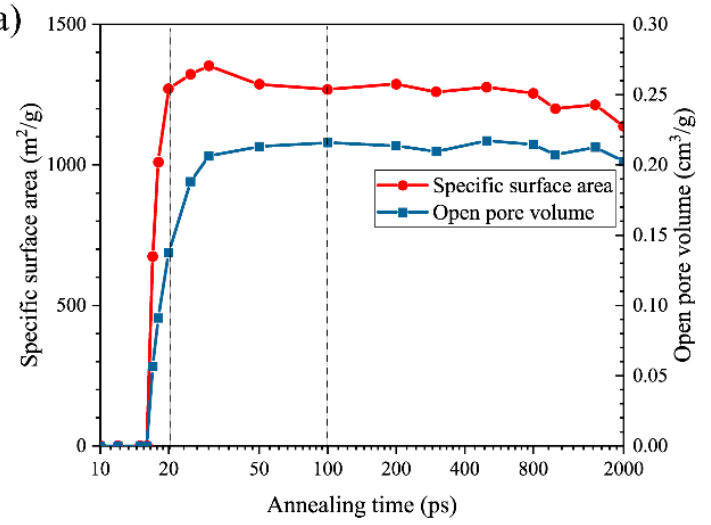

(c) $10 \mathrm{ps}$

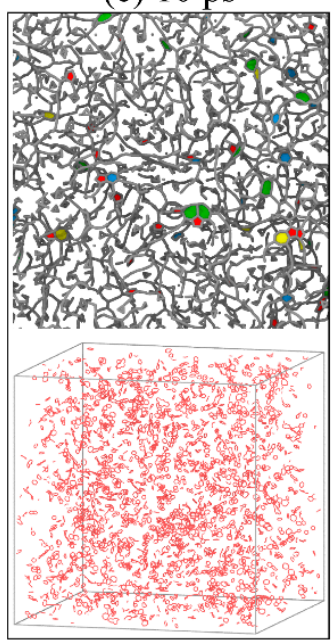

(d) $20 \mathrm{ps}$

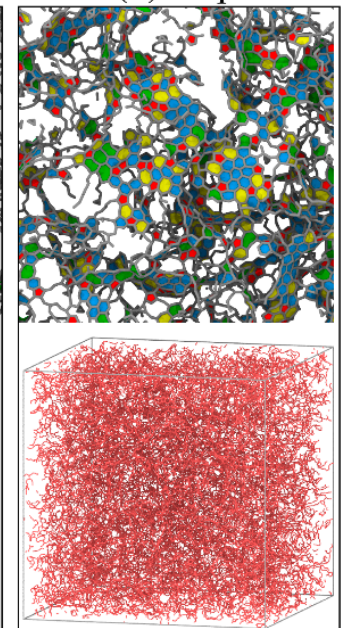

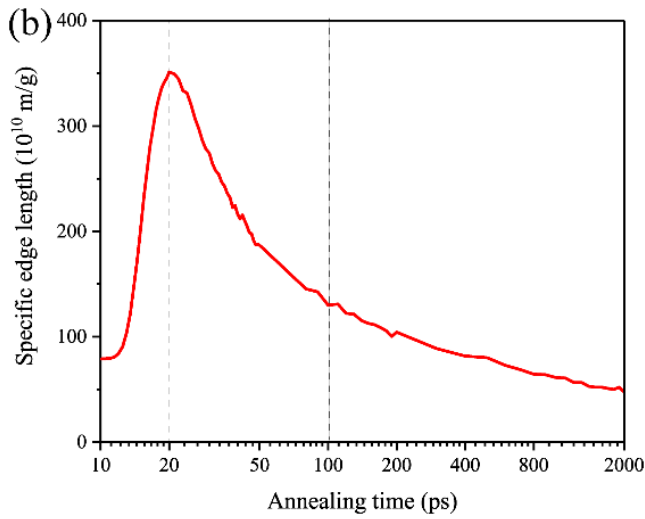

(e) $50 \mathrm{ps}$

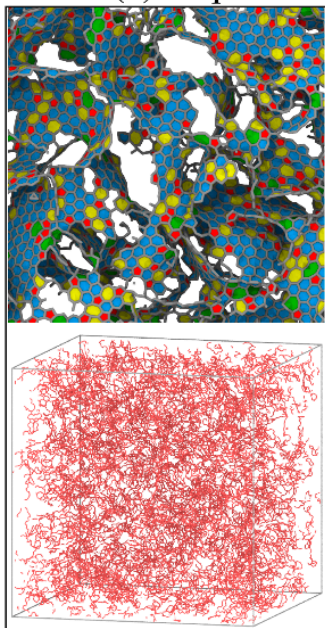

(f) $2000 \mathrm{ps}$

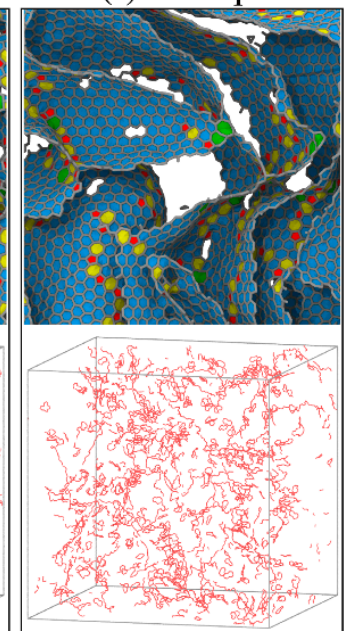

Figure 1. (a) Evolution of specific surface area and open pore volume during the annealing process. (b) The specific edge length of fragments as a function of annealing time. (c-f) Snapshots of local pores (upper) and the configuration of edges (below) at several annealing points. Rings are distinguished by different colors (pentagon in red, hexagon in blue, heptagon in yellow, octagon in green).

In the last stage (from 100 ps to 2000 ps), the framework underwent further graphitization. A large portion of nonhexagonal rings transformed into 6-membered rings. In the final structure, the number of hexagonal rings is 0.443 per atom, near 0.5 in infinite graphene. According to the Arrhenius framework, the model simulated at $4000 \mathrm{~K}$ for $2 \mathrm{~ns}$ has the same reaction probability with the one annealed at $1200{ }^{\circ} \mathrm{C}$ for $10^{4} \mathrm{~s}$. The $s p^{2}$ fractions in the model annealed at $4000 \mathrm{~K}$ accounted for $97.1 \%$, which is close to that of CDCs prepared at $1200{ }^{\circ} \mathrm{C}$ for hours in experiments [46], indicating the strength of the simulation approach to mimic experimental results. We can tell that the structure finally transformed into a network of disordered graphene layers. During this stage, the free edges continued to decline because of the merging of layers and healing of defects. The change of pore structure was not significant since the main framework was already established in the former stage.

The surface area and pore volume do not include all the information we need about pore structure. The pore size is also one of the main factors influencing adsorption and mass diffusion in the material. In experiments, pore size can be estimated indirectly from experimental isotherms for gas adsorption. From the computational point of view, we can compute the PSD function from the coordinates of carbon atoms. The computed PSD at several annealing times are presented in Figure 2a. We found that the PSD became wider over annealing time. At $20 \mathrm{ps}$, the maximum diameter was $1.15 \mathrm{~nm}$. It increased to $1.71 \mathrm{~nm}$ at $100 \mathrm{ps}$. The average pore size, calculated from the PSD, also increased dramatically from 20 to 100 ps. From the rendered pore pictures in Figure $2 b-d$, we observed the formation 
and growth of pores. At the beginning, the open pores formed through the connecting of initially segregated closed pores during the first stage, and the size was even and small. As the graphene-like framework developed during the second stage, the pore size became bigger, and slit pores were found in the interlayer space of stacked structures. The PSD and rendered pore structure in Figure 2e show little change in the last stage, during which the average pore size changed slightly from $0.93 \mathrm{~nm}$ to $0.96 \mathrm{~nm}$. After annealing, there are some free atoms and gaseous triangles, involving about $0.16 \%$ of the initial atoms. These atoms were removed from the system. After energy minimization, the average pore size of the final model is $1.09 \mathrm{~nm}$, and the maximum pore size is $2.19 \mathrm{~nm}$. Experimentally, the PSD is calculated from adsorption isotherms. The experimental average pore size of the DGN annealed at $1200{ }^{\circ} \mathrm{C}$ is $1.28 \mathrm{~nm}$ [46], slightly larger than our results. A minor portion of 2.0-4.0 nm sized mesopores were found in experiments. To describe the minor portion of mesopores in simulations, a much larger system size is needed, which is beyond our computing capability.

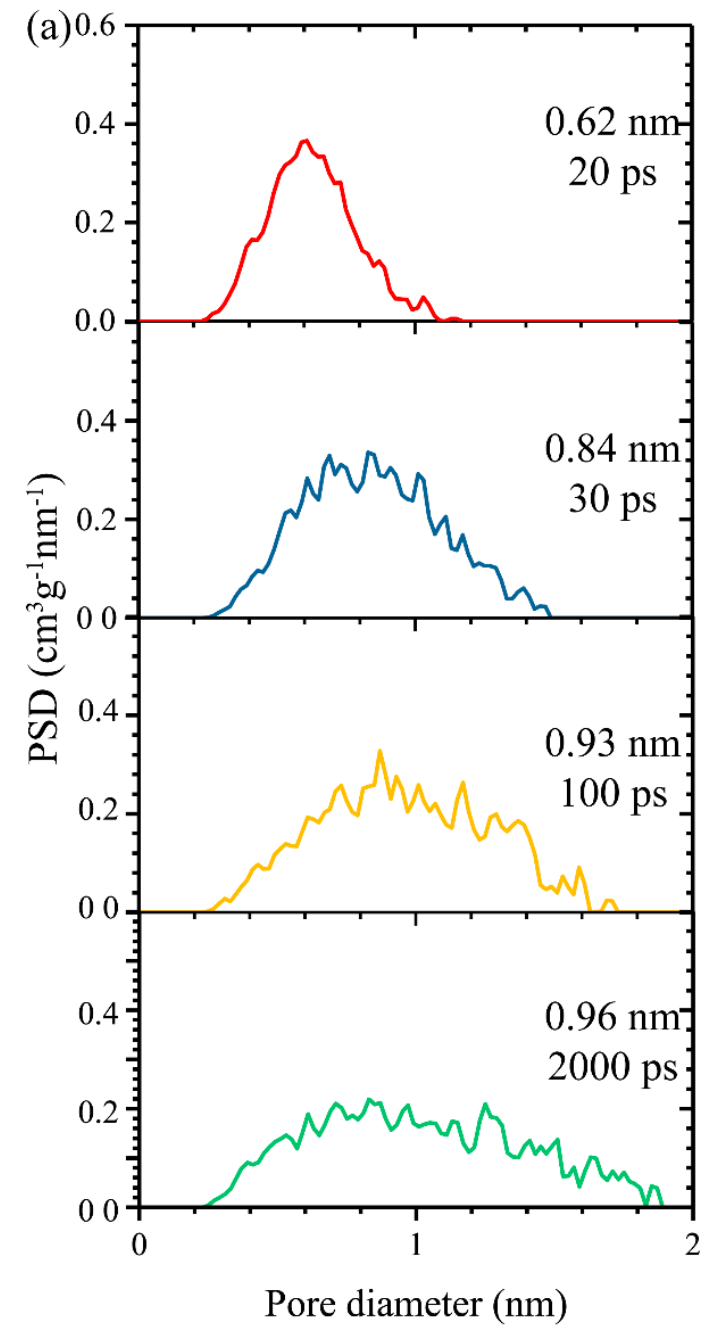

(b)

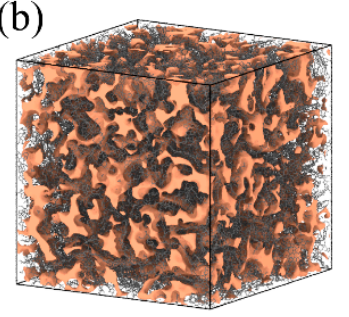

(c)

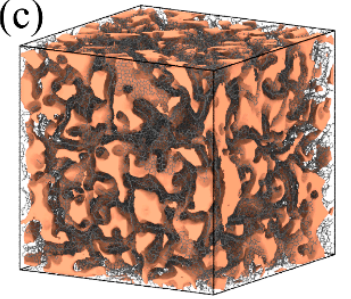

(d)

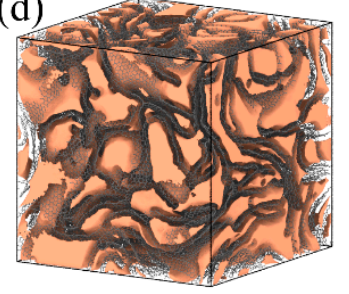

(e)

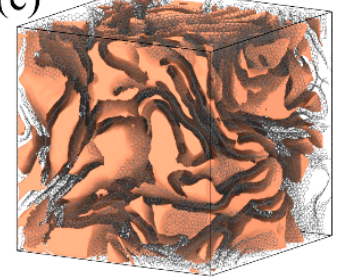

Figure 2. (a) Pore size distribution function at several annealing time shown in different panels. The average size of pores is shown in the corresponding panel. (b-e) The open pore structure at each time was rendered and displayed on the right.

From the above results, we know that the pores in the annealed disordered graphene networks have complicated shapes because of the curving and stacking of graphene layers. Furthermore, the porous structures are determined at the very early annealing stage. During the most prolonged stage, the pore size and volume show little change. We can estimate the corresponding experimental time of each stage. The simulation at $4000 \mathrm{~K}$ for 
$100 \mathrm{ps}$ can be related to $1200^{\circ} \mathrm{C}$ for $500 \mathrm{~s}$ based on the Arrhenius framework [31]. However, the real experimental time needed for forming the framework will be longer than $500 \mathrm{~s}$ because it takes additional time to remove noncarbon atoms from the matrix.

Then we investigate the influence of density on the porous properties of DGNs. The initial density of carbon atoms ranging from $0.1 \mathrm{~g} / \mathrm{cm}^{3}$ to $2.3 \mathrm{~g} / \mathrm{cm}^{3}$ was controlled by changing the box size during the first 10 ps when the number of carbon atoms was 108,000. Because of the changes in box size during relaxation, the final density is different from the initial density. So, we only refer to the final density when mentioned.

The level of graphitization can be characterized by the fraction of $s p^{2}$ atoms. For infinite graphene, $s p^{2}$ atoms account for $100 \%$. In our simulations, the models at all densities show the same high degree of graphitization when the annealing temperature was $4000 \mathrm{~K}$. As shown in Table 1, the fractions of $s p^{2}$ atoms are around $98 \%$ from the lowest density $0.10 \mathrm{~g} / \mathrm{cm}^{3}$ to the highest density $1.64 \mathrm{~g} / \mathrm{cm}^{3}$. The carbon rings in all models are dominated by hexagons, and each atom shares 0.45 hexagons on average, which is close to the limit of infinite graphene, 0.5 . The difference comes from a small amount of nonhexagonal rings and edges of limited-size graphene layers. We also calculated the total edge length of all models. The specific length sizes are similar, around $20 \times 10^{10} \mathrm{~m} / \mathrm{g}$. In 2011, López studied the porosity structure of a carbon system based on Tersoff potential [27]. Their models annealed at high temperature have similar specific edge lengths with our results.

Table 1. Fraction of $s p^{2}$ atoms and specific edge length of several models with different densities.

\begin{tabular}{ccc}
\hline Density $\left(\mathbf{g} / \mathbf{c m}^{\mathbf{3}}\right)$ & $\boldsymbol{s} \boldsymbol{p}^{\mathbf{2}}$ Fraction $(\mathbf{\%})$ & Specific Edge Length $(\times \mathbf{1 0} \mathbf{1 0} \mathbf{~} / \mathbf{g})$ \\
\hline 0.10 & 98.3 & 19.4 \\
0.56 & 98.0 & 20.0 \\
1.01 & 97.9 & 19.3 \\
1.64 & 98.0 & 19.6 \\
\hline
\end{tabular}

To compare the porous properties at different densities, we calculated the ratio of porosity. As shown in Figure 3a, the total porosity decreases almost linearly with the increase of density. This tendency is easy to explain. From the definition of total porosity, it can be related to a function of the density of disordered graphene networks, $\rho$, and the density of dense carbon material, $\rho_{0}$ :

$$
p_{\text {total }}=\frac{V_{\text {pore }}}{V_{\text {total }}}=1-\frac{\rho}{\rho_{0}}
$$
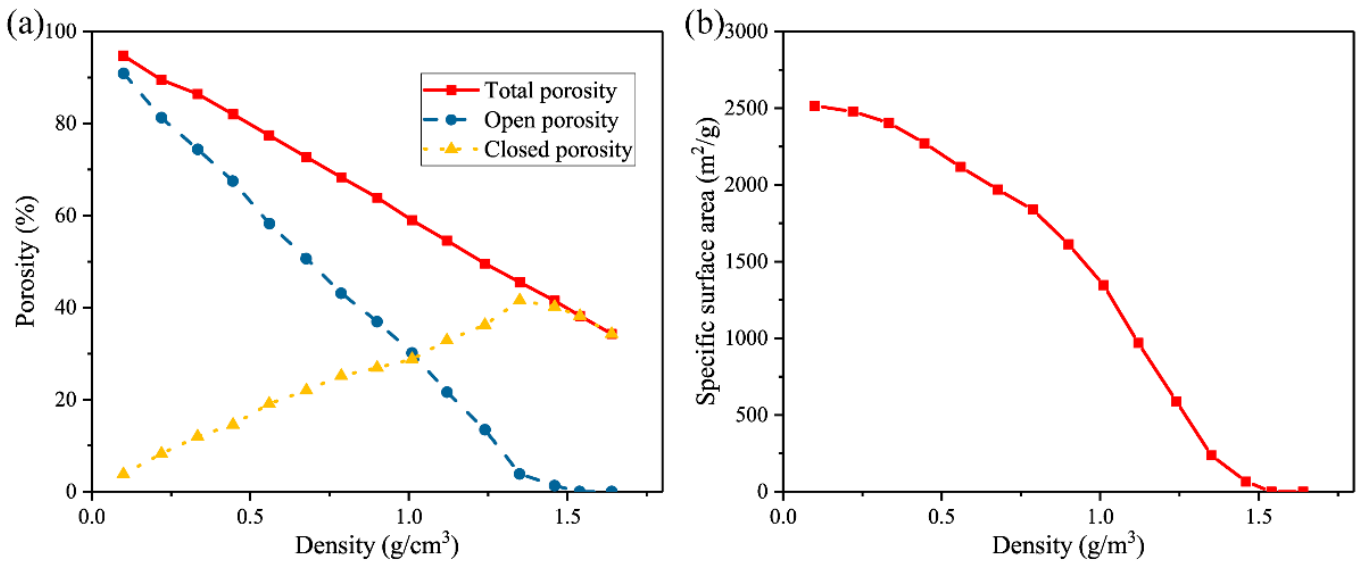

Figure 3. (a) Ratio of porosities as a function of density. (b) Specific surface area declines with increasing density.

This equation directly shows the linear relationship between the total porosity and the density of the material. By linearly fitting the data of total porosity in Figure 3a, the 
intercept is $99.15 \%$, which agrees well with the equation, indicating the rationality of the method to calculate the pore volume. The fitted $\rho_{0}$ is $2.50 \mathrm{~g} / \mathrm{cm}^{3}$, corresponding to the density of single-layered graphene. It should be noted that this density is based on the sphere model of atoms, which includes less volume than the membrane model with a thickness of $3.4 \AA$. So, the fitted $\rho_{0}$ is larger than the often used density of crystalline graphite, $2.267 \mathrm{~g} / \mathrm{cm}^{3}$.

We also calculated the ratio of open porosity and closed porosity by percolation analysis. At the very low density of $0.1 \mathrm{~g} / \mathrm{cm}^{3}$, nearly all pores are open because there is no stacking of graphene layers, enabling the connection and opening of all void space. When the density increases, stacking of graphene layers forms, and some interlayer voids become inaccessible. So, the open porosity decreases faster than total porosity. At the same time, closed porosity increases. When density is larger than $1.0 \mathrm{~g} / \mathrm{cm}^{3}$, graphene layers are large enough to divide the pores into segregated spaces, and some spaces are wrapped and isolated from other open pores. When density reached $1.35 \mathrm{~g} / \mathrm{cm}^{3}$, open porosity drops to $3.9 \%$, even though the total porosity is $33.4 \%$. So, the permeability of these disordered graphene networks is weak. As density continues to increase from $1.35 \mathrm{~g} / \mathrm{cm}^{3}$, all types of porosity decline, and nearly all of the pores are closed.

In porous carbon materials, the SSA is highly dependent on the porosity. Figure $3 \mathrm{~b}$ shows that the SSA of DGNs declines monotonically with the increase of density. When density is lower than $0.5 \mathrm{~g} / \mathrm{cm}^{3}$, the SSA is above $2100 \mathrm{~m}^{2} / \mathrm{g}$, and we find a saturation of the SSA. Given the same mass of disordered graphene networks and the same graphitization level, when the density is low enough, nearly all pores are open, so the accessible surface areas of different models are saturated to the theoretical SSA of single-layered graphene. Further reduction of density does not result in a significant increase in the SSA. At densities higher than $1.35 \mathrm{~g} / \mathrm{cm}^{3}$, even though there are pores in the material, the SSA is near zero because few pores are accessible.

The rendered images of pores, including total pores and open pores, are presented in Figure 4c. For low-density models, pores are connected in the whole space, and open pores have the same shape with total pores. While at higher densities, the pores are segregated by walls of graphene layers, and only part of them are open. The intensity and distribution range of PSDs for different densities show a huge difference, as shown in Figure $4 \mathrm{a}$. We obtained the maximum pore size and average pore size from the PSD value. They both decreased with increasing density. The average pore size can range from $1 \mathrm{~nm}$ to more than $10 \mathrm{~nm}$. So, the pore size is tunable by adjusting the density of disordered graphene networks.

Another critical factor that influences the structure of DGNs is the annealing temperature. We performed a series of annealing-MD simulations by changing the target annealing temperature from 1000 to $4000 \mathrm{~K}$ while kept the initial densities as $1.0 \mathrm{~g} / \mathrm{cm}^{3}$. The temperature settings can be related to experimental temperatures from 400 to $1200{ }^{\circ} \mathrm{C}$. After the same relaxation and energy minimization process, we get DNGs annealed at different temperatures. We find that annealing temperature has a significant influence on the graphitization level. The $s p^{2}$ fraction increases from $74 \%$ at $1000 \mathrm{~K}$ to $98 \%$ at $4000 \mathrm{~K}$, indicating the DGNs annealed at low-temperatures are not highly graphitized. In low-temperature models, carbon atoms form a network of small fragments with many free edges, which is different from the graphenelike layers in high-temperature models. Figure 5a shows that all DGNs have a similar total porosity, while the open porosity for $2500 \mathrm{~K}$ (corresponding to $800 \sim 1000{ }^{\circ} \mathrm{C}$ in experiments) is higher than other models. This result is consistent with Dash's experimental study on CDCs from Titanium carbide, where they reported that the micropore volumes at 800 and $1000{ }^{\circ} \mathrm{C}$ of are larger than other temperatures [23]. In Figure 5b, the SSA decreases dramatically with the increasing annealing temperature, and the tendency is different from the porosity, indicating that free edges have a more significant influence than the porosity in these models. In Gläsel's work on CDCs [46], the experimental SSA also decreases with increasing temperature. 

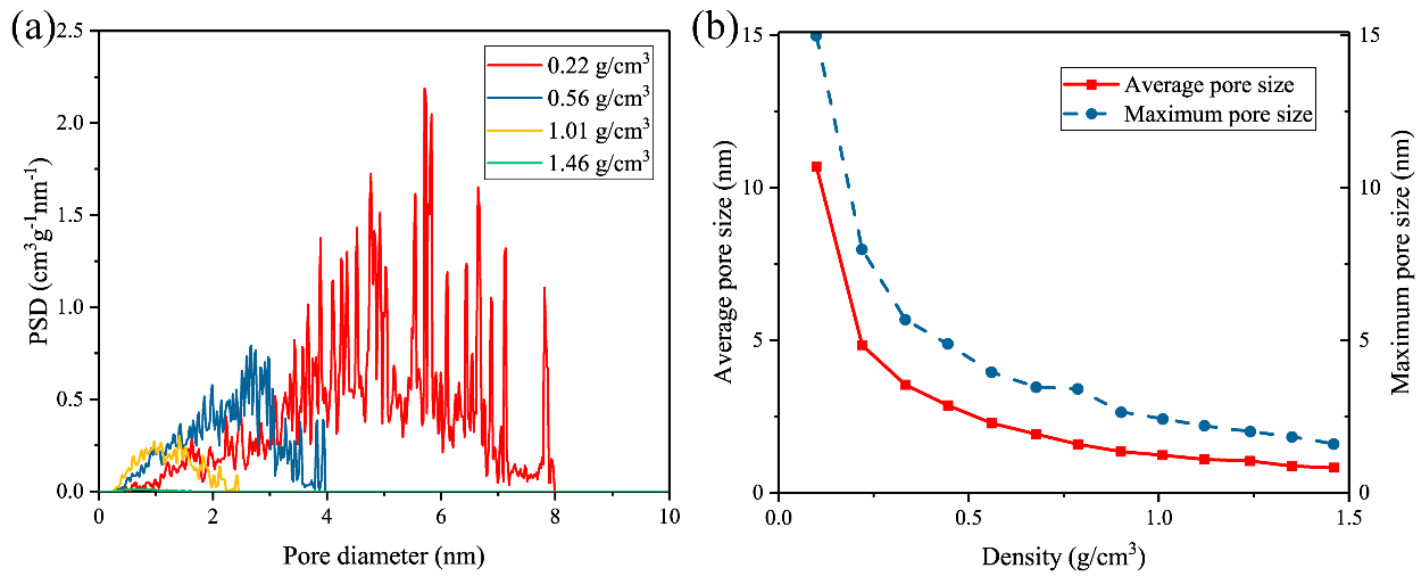

(c) $0.22 \mathrm{~g} / \mathrm{cm}^{3}$
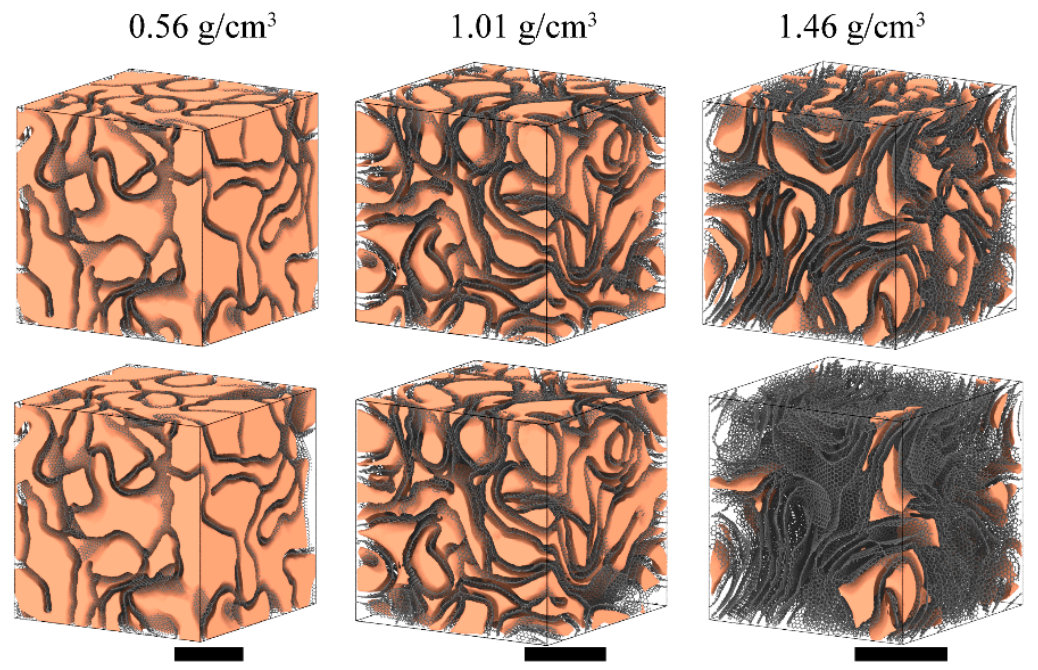

Figure 4. (a) Pore size distribution function at different densities. (b) Average pore size and maximum pore size obtained from the pore size distribution (PSD) as a function of density. (c) Rendered images of total and open pore structures for several densities. The black scale bars represent $5 \mathrm{~nm}$.

The calculated PSD for DGNs annealed at different temperatures are shown in Figure 6a. When the annealing temperature rises, the distribution of pore size becomes broader. At the same time, the position of the peak, which represents the most accessible pore size, becomes larger. The average pore size is calculated and presented in each panel. It increased from $0.71 \mathrm{~nm}$ to $1.09 \mathrm{~nm}$. The increasing pore size can be seen from the rendered images of each DGNs, as is shown in Figure $6 \mathrm{~b}-\mathrm{e}$. At low temperatures, the pores are uniform, separated by walls of small fragments. While at high temperatures, space is divided by graphene layers, and big slit pores are found. The PSD of our simulations agrees with the experimental results of CDCs [15], where the PSD of ZrC-CDCs shows increasing pore size with the increase of temperature from 600 to $1200{ }^{\circ} \mathrm{C}$. Experimental results suggested that highly graphitized carbon materials adsorbed less hydrogen than less graphitized carbons [24]. Our simulations reveal that smaller pore size results in larger SSA given the same pore volume. Therefore, one can adjust the pore size to achieve better gas adsorption performance by changing the annealing temperature during material processing. 
(a)
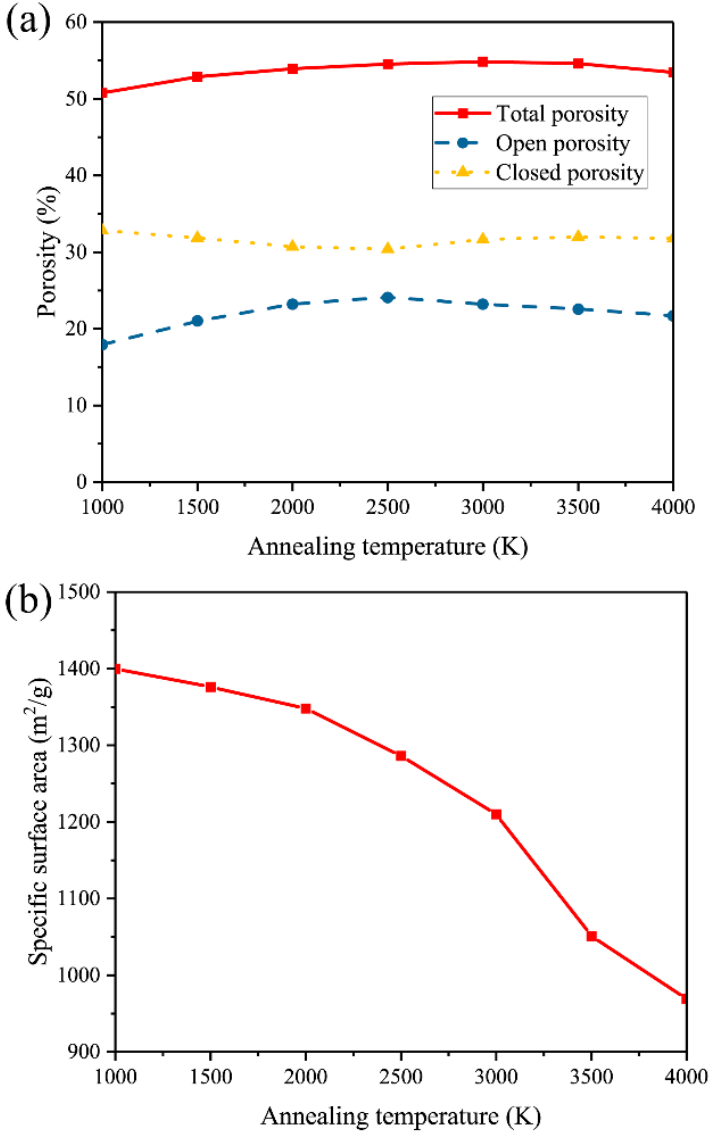

Figure 5. The porosity (a) and specific surface area (b) of disordered graphene networks (DGNs) annealed at different temperatures.

\section{Conclusions}

In this work, we have applied MD simulations to investigate the porous characteristics of DGNs using large models containing over 100,000 atoms. The DGNs of varying densities were annealed at different temperatures for $2 \mathrm{~ns}$, and the annealing temperature and time can be related to experimental conditions using an Arrhenius framework. To understand how porous structures form and evolve, we chose the DGN of $1.0 \mathrm{~g} / \mathrm{cm}^{3}$ annealed at $4000 \mathrm{~K}$ as the representative and calculated the porous properties at different annealing times. In the formation of polyaromatic fragments, the volume of small open pores and SSA increased dramatically. These polyaromatic fragments then merge into larger layers and form a framework for DGNs, during which open pores connect and evolve into larger pores with a slit feature, and the SSA decrease because of the reduction of free edges. In the rest time of annealing, the porous properties show little changes.

Then, the influence of densities on the porous structures of DGNs was investigated. The ratio of porosity, pore size, and SSA increase with decreasing density. For highdensity DGNs, the pores are confined in the interlayer spaces because of the stacking of graphene layers, resulting in closed and slit pores. For low-density DGNs, there is no stacking structure, and the pores are well interconnected. We also observed a saturation phenomenon of SSA with decreasing density, which comes from a similar graphitization level and edge lengths. Finally, we investigated the porous structures of DGNs annealed at different temperatures. At low temperatures, the pores are small, and the walls are small polyaromatic fragments, which results in higher SSA than highly graphitized DGNs at high temperatures. Our results suggest that the pore size in DNGs can be adjusted by carefully choosing the density and annealing temperature, which would guide the design and procession of nanoporous carbon materials. 
(a)

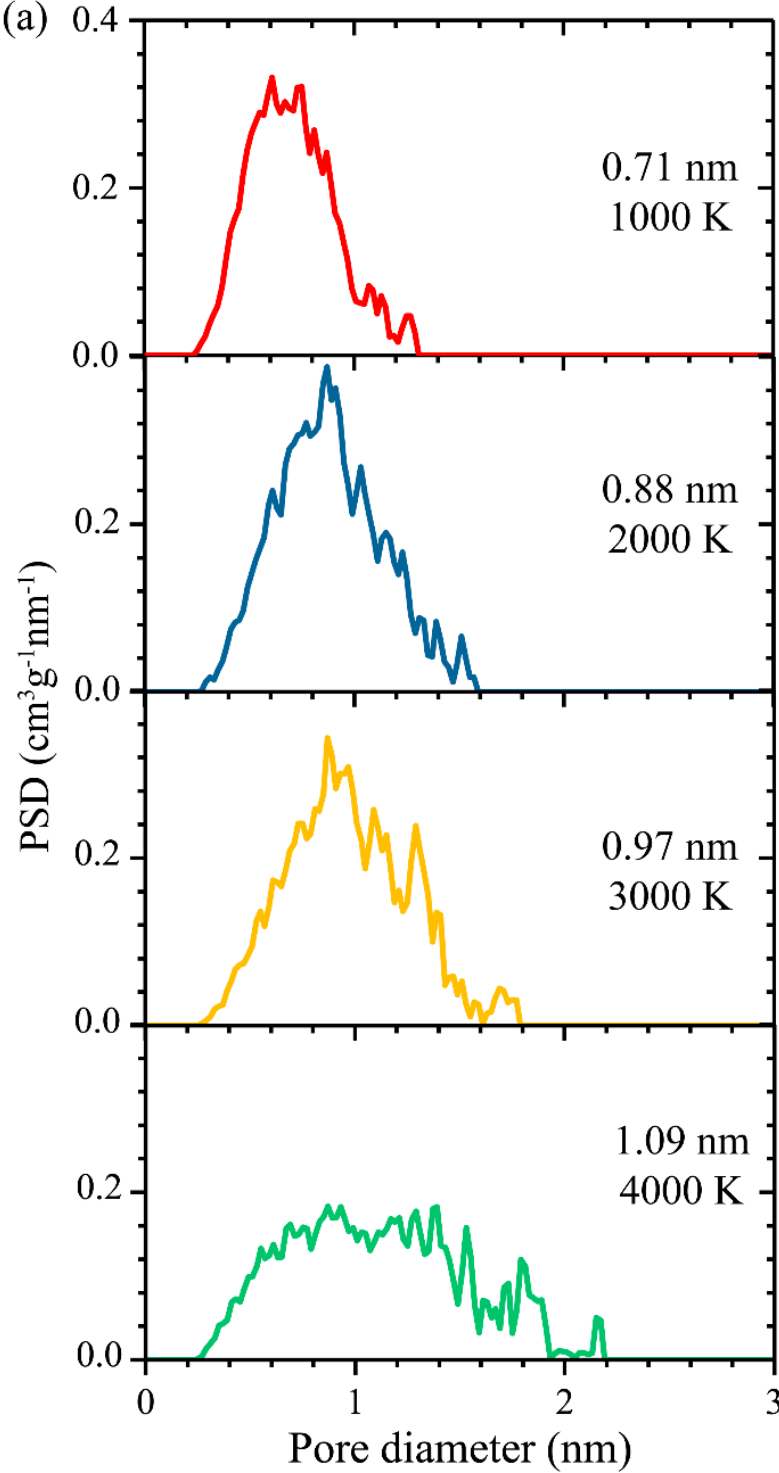

(b)

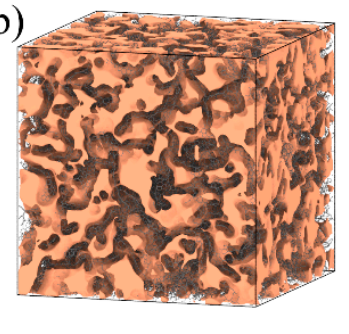

(c)

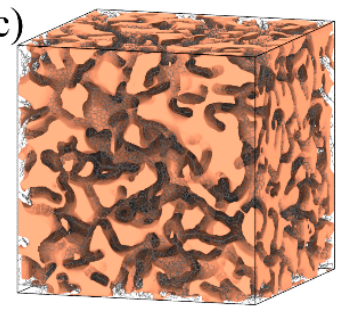

(d)

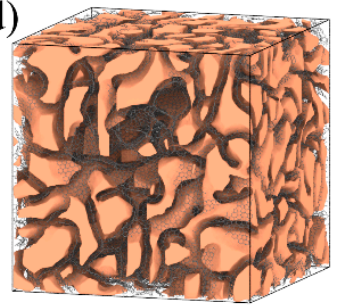

(e)

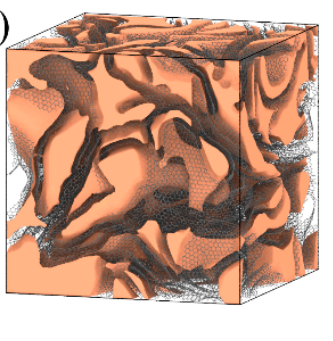

$5 \mathrm{~nm}$

Figure 6. (a) Pore size distributions for several DGNs annealed at different temperatures. The average pore size is shown in each panel. The corresponding rendered images of open pores are in $(\mathbf{b}-\mathbf{e})$.

Author Contributions: Y.W.: Investigation, Methodology, Software, Data Curation, Formal analysis, Writing-Original Draft. Y.Z.: Conceptualization, Formal analysis, Validation, Visualization, Supervision, Funding acquisition, Writing-Review and Editing. H.W.: Supervision, Project administration, Resources, Funding acquisition. All authors have read and agreed to the published version of the manuscript.

Funding: This research was funded by the Strategic Priority Research Program of the Chinese Academy of Sciences (XDB22040402), the National Natural Science Foundation of China (11872063 and 11802302), and the USTC Research Funds of the Double First-Class Initiative (YD2480002002).

Institutional Review Board Statement: Not applicable.

Informed Consent Statement: Not applicable.

Data Availability Statement: Data is contained within the article.

Acknowledgments: We thank Nigel A. Marks at Curtin University for providing EDIP potential. This work was jointly supported by the Strategic Priority Research Program of the Chinese Academy of Sciences (XDB22040402), the National Natural Science Foundation of China (11872063 and 11802302), and the USTC Research Funds of the Double First-Class Initiative (YD2480002002). The numeri- 
cal calculations have been done on the supercomputing system in the Supercomputing Center of University of Science and Technology of China.

Conflicts of Interest: The authors declare no conflict of interest.

\section{References}

1. Sethia, G.; Sayari, A. Activated carbon with optimum pore size distribution for hydrogen storage. Carbon 2016, 99, 289-294. [CrossRef]

2. Tin, P.S.; Chung, T.-S.; Liu, Y.; Wang, R. Separation of $\mathrm{CO}_{2} / \mathrm{CH}_{4}$ through carbon molecular sieve membranes derived from P84 polyimide. Carbon 2004, 42, 3123-3131. [CrossRef]

3. Lu, C.; Tranca, D.; Zhang, J.; Rodriguez Hernandez, F.; Su, Y.; Zhuang, X.; Zhang, F.; Seifert, G.; Feng, X. Molybdenum carbideembedded nitrogen-doped porous carbon nanosheets as electrocatalysts for water splitting in alkaline media. ACS Nano 2017, 11, 3933-3942. [CrossRef] [PubMed]

4. Wei, T.; Wei, X.; Gao, Y.; Li, H. Large scale production of biomass-derived nitrogen-doped porous carbon materials for supercapacitors. Electrochim. Acta 2015, 169, 186-194. [CrossRef]

5. Novoselov, K.S.; Geim, A.K.; Morozov, S.V.; Jiang, D.; Zhang, Y.; Dubonos, S.V.; Grigorieva, I.V.; Firsov, A.A. Electric field effect in atomically thin carbon films. Science 2004, 306, 666-669. [CrossRef]

6. Iijima, S. Helical microtubules of graphitic carbon. Nature 1991, 354, 56-58. [CrossRef]

7. Mintmire, J.W.; Dunlap, B.I.; White, C.T. Are fullerene tubules metallic? Phys. Rev. Lett. 1992, 68, 631. [CrossRef]

8. Zaman, N.; Malik, R.A.; Alrobei, H.; Kim, J.; Latif, M.; Hussain, A.; Maqbool, A.; Karim, R.A.; Saleem, M.; Asif Rafiq, M.; et al. Structural and Electrochemical Analysis of Decarburized Graphene Electrodes for Supercapacitor Applications. Crystals 2020, 10, 1043. [CrossRef]

9. Caturla, F.; Molina-Sabio, M.; Rodriguez-Reinoso, F. Preparation of activated carbon by chemical activation with $\mathrm{ZnCl}_{2}$. Carbon 1991, 29, 999-1007. [CrossRef]

10. Gogotsi, Y.; Nikitin, A.; Ye, H.; Zhou, W.; Fischer, J.E.; Yi, B.; Foley, H.C.; Barsoum, M.W. Nanoporous carbide-derived carbon with tunable pore size. Nat. Mater. 2003, 2, 591-594. [CrossRef]

11. Luong, D.X.; Bets, K.V.; Algozeeb, W.A.; Stanford, M.G.; Kittrell, C.; Chen, W.; Salvatierra, R.V.; Ren, M.; McHugh, E.A.; Advincula, P.A.; et al. Gram-scale bottom-up flash graphene synthesis. Nature 2020, 577, 647-651. [CrossRef] [PubMed]

12. Chen, M.; Zhu, Y.; Xia, J.; Wu, H. Molecular insights into the initial formation of pyrolytic carbon upon carbon fiber surface. Carbon 2019, 148, 307-316. [CrossRef]

13. Shafeeyan, M.S.; Daud, W.M.A.W.; Houshmand, A.; Shamiri, A. A review on surface modification of activated carbon for carbon dioxide adsorption. J. Anal. Appl. Pyrolysis 2010, 89, 143-151. [CrossRef]

14. Dash, R.K.; Yushin, G.; Gogotsi, Y. Synthesis, structure and porosity analysis of microporous and mesoporous carbon derived from zirconium carbide. Microporous Mesoporous Mater. 2005, 86, 50-57. [CrossRef]

15. Gogotsi, Y.; Dash, R.K.; Yushin, G.; Yildirim, T.; Laudisio, G.; Fischer, J.E. Tailoring of nanoscale porosity in carbide-derived carbons for hydrogen storage. J. Am. Chem. Soc. 2005, 127, 16006-16007. [CrossRef]

16. Stanford, M.G.; Bets, K.V.; Luong, D.X.; Advincula, P.A.; Chen, W.; Li, J.T.; Wang, Z.; McHugh, E.A.; Algozeeb, W.A.; Yakobson, B.I.; et al. Flash Graphene Morphologies. ACS Nano 2020, 14, 13691-13699. [CrossRef]

17. Jenkins, G.M.; Kawamura, K. Structure of glassy carbon. Nature 1971, 231, 175-176. [CrossRef]

18. Harris, P.J.F.; Tsang, S.C. High-resolution electron microscopy studies of non-graphitizing carbons. Philos. Mag. A 1997, 76, 667-677. [CrossRef]

19. Jeanne-Rose, V.; Golabkan, V.; Mansot, J.L.; Largitte, L.; Césaire, T.; Ouensanga, A. An EELS-based study of the effects of pyrolysis on natural carbonaceous materials used for activated charcoal preparation. J. Microsc. 2003, 210, 53-59. [CrossRef]

20. Schimmel, H.G.; Kearley, G.J.; Nijkamp, M.G.; Visser, C.T.; de Jong, K.P.; Mulder, F.M. Hydrogen adsorption in carbon nanostructures: Comparison of nanotubes, fibers, and coals. Chem. Eur. J. 2003, 9, 4764-4770. [CrossRef]

21. Sevilla, M.; Mokaya, R. Energy storage applications of activated carbons: Supercapacitors and hydrogen storage. Energy Environ. Sci. 2014, 7, 1250-1280. [CrossRef]

22. Opletal, G.; Petersen, T.; O’Malley, B.; Snook, I.; McCulloch, D.G.; Marks, N.A.; Yarovsky, I. Hybrid approach for generating realistic amorphous carbon structure using metropolis and reverse Monte Carlo. Mol. Simul. 2002, 28, 927-938. [CrossRef]

23. Dash, R.; Chmiola, J.; Yushin, G.; Gogotsi, Y.; Laudisio, G.; Singer, J.; Fischer, J.; Kucheyev, S. Titanium carbide derived nanoporous carbon for energy-related applications. Carbon 2006, 44, 2489-2497. [CrossRef]

24. Jiménez, V.; Ramirez-Lucas, A.; Sánchez, P.; Valverde, J.L.; Romero, A. Hydrogen storage in different carbon materials: Influence of the porosity development by chemical activation. Appl. Surf. Sci. 2012, 258, 2498-2509. [CrossRef]

25. Farmahini, A.H.; Bhatia, S.K. Effect of structural anisotropy and pore-network accessibility on fluid transport in nanoporous $\mathrm{Ti}_{3} \mathrm{SiC}_{2}$ carbide-derived carbon. Carbon 2016, 103, 16-27. [CrossRef]

26. Farmahini, A.H.; Bhatia, S.K. Hybrid Reverse Monte Carlo simulation of amorphous carbon: Distinguishing between competing structures obtained using different modeling protocols. Carbon 2015, 83, 53-70. [CrossRef]

27. López, M.J.; Cabria, I.; Alonso, J.A. Simulated porosity and electronic structure of nanoporous carbons. J. Chem. Phys. 2011, 135, 104706. [CrossRef] [PubMed]

28. Mejia-Mendoza, L.M.; Valdez-Gonzalez, M.; Muñiz, J.; Santiago, U.; Cuentas-Gallegos, A.K.; Robles, M. A theoretical approach to the nanoporous phase diagram of carbon. Carbon 2017, 120, 233-243. [CrossRef] 
29. Schweizer, S.; Meißner, R.; Amkreutz, M.; Thiel, K.; Schiffels, P.; Landwehr, J.; Etzold, B.J.M.; Hill, J.-R. Molecular modeling of microporous structures of carbide-derived carbon-based supercapacitors. J. Phys. Chem. C 2017, 121, 7221-7231. [CrossRef]

30. Palmer, J.C.; Llobet, A.; Yeon, S.-H.; Fischer, J.E.; Shi, Y.; Gogotsi, Y.; Gubbins, K.E. Modeling the structural evolution of carbide-derived carbons using quenched molecular dynamics. Carbon 2010, 48, 1116-1123. [CrossRef]

31. de Tomas, C.; Suarez-Martinez, I.; Vallejos-Burgos, F.; López, M.J.; Kaneko, K.; Marks, N.A. Structural prediction of graphitization and porosity in carbide-derived carbons. Carbon 2017, 119, 1-9. [CrossRef]

32. Martin, J.W.; de Tomas, C.; Suarez-Martinez, I.; Kraft, M.; Marks, N.A. Topology of disordered 3D graphene networks. Phys. Rev. Lett. 2019, 123, 116105. [CrossRef] [PubMed]

33. Marks, N.A. Generalizing the environment-dependent interaction potential for carbon. Phys. Rev. B 2000, 63, 35401. [CrossRef]

34. de Tomas, C.; Suarez-Martinez, I.; Marks, N.A. Graphitization of amorphous carbons: A comparative study of interatomic potentials. Carbon 2016, 109, 681-693. [CrossRef]

35. Yushin, G.; Dash, R.; Jagiello, J.; Fischer, J.E.; Gogotsi, Y. Carbide-derived carbons: Effect of pore size on hydrogen uptake and heat of adsorption. Adv. Funct. Mater. 2006, 16, 2288-2293. [CrossRef]

36. Stuart, S.J.; Tutein, A.B.; Harrison, J.A. A reactive potential for hydrocarbons with intermolecular interactions. J. Chem. Phys. 2000, 112, 6472-6486. [CrossRef]

37. Zhan, H.; Zhang, Y.; Yang, C.; Zhang, G.; Gu, Y. Graphene helicoid as novel nanospring. Carbon 2017, 120, 258-264. [CrossRef]

38. Han, X.; Xu, F.; Duan, S.; Zhan, H.; Gu, Y.; Liu, G. A novel super-elastic carbon nanofiber with cup-stacked carbon nanocones and a screw dislocation. Carbon 2019, 154, 98-107. [CrossRef]

39. Wang, Y.; Zhu, Y.; Wang, F.; Liu, X.; Wu, H. Super-elasticity and deformation mechanism of three-dimensional pillared graphene network structures. Carbon 2017, 118, 588-596. [CrossRef]

40. Zhan, H.; Zhang, G.; Bell, J.M.; Gu, Y. The morphology and temperature dependent tensile properties of diamond nanothreads. Carbon 2016, 107, 304-309. [CrossRef]

41. Plimpton, S. Fast parallel algorithms for short-range molecular dynamics. J. Comput. Phys. 1995, 117, 1-19. [CrossRef]

42. Franzblau, D.S. Computation of ring statistics for network models of solids. Phys. Rev. B 1991, 44, 4925. [CrossRef] [PubMed]

43. Stukowski, A. Visualization and analysis of atomistic simulation data with OVITO-The Open Visualization Tool. Model. Simul. Mater. Sci. Eng. 2009, 18, 15012. [CrossRef]

44. Sarkisov, L.; Harrison, A. Computational structure characterisation tools in application to ordered and disordered porous materials. Mol. Simul. 2011, 37, 1248-1257. [CrossRef]

45. Cheng, H.; Cooper, A.C.; Pez, G.P.; Kostov, M.K.; Piotrowski, P.; Stuart, S.J. Molecular dynamics simulations on the effects of diameter and chirality on hydrogen adsorption in single walled carbon nanotubes. J. Phys. Chem. B 2005, 109, 3780-3786. [CrossRef]

46. Gläsel, J.; Diao, J.; Feng, Z.; Hilgart, M.; Wolker, T.; Su, D.S.; Etzold, B.J.M. Mesoporous and graphitic carbide-derived carbons as selective and stable catalysts for the dehydrogenation reaction. Chem. Mater. 2015, 27, 5719-5725. [CrossRef] 